\title{
The first outburst of SAX J1808.4-3658 revisited
}

\author{
J. J. M. in 't Zand ${ }^{1,2}$, R. Cornelisse ${ }^{1,2}$, E. Kuulkers ${ }^{1,2}$, J. Heise ${ }^{1}$, L. Kuiper $^{1}$, A. Bazzano ${ }^{3}$, M. Cocchi ${ }^{3}$, \\ J. M. Muller ${ }^{1,4, \star}$, L. Natalucci ${ }^{3}$, M. J. S. Smith ${ }^{1,5, \star \star}$, and P. Ubertini ${ }^{3}$
}

1 Space Research Organization Netherlands, Sorbonnelaan 2, 3584 CA Utrecht, The Netherlands

2 Astronomical Institute, Utrecht University, PO Box 80000, 3508 TA Utrecht, The Netherlands

3 Istituto di Astrofisica Spaziale (CNR), Area Ricerca Roma Tor Vergata, Via del Fosso del Cavaliere, 00133 Roma, Italy

4 BeppoSAX Science Data Center, Nuova Telespazio, Via Corcolle 19, 00131 Roma, Italy

5 BeppoSAX Science Operation Center, Nuova Telespazio, Via Corcolle 19, 00131 Roma, Italy

Received 30 August 2000 / Accepted 6 April 2001

\begin{abstract}
Data of the 1996 outburst of the single-known accreting millisecond pulsar SAX J1808.4-3658, taken with the Wide Field Cameras (WFCs) on BeppoSAX, are revisited with more complete data coverage and more comprehensive analysis techniques than in a previous report. An additional type-I X-ray burst was identified which occurred at a time when the persistent emission is below the detection limit, roughly 30 days after outburst maximum. This burst is three times longer than the first two bursts, and $50 \%$ brighter. It is the brightest burst within the $\sim 1700$ type-I bursts detected so far with the WFCs. A spectral analysis of the data reveals a distance to SAX J1808.4-3658 of $\sim 2.5 \mathrm{kpc}$. This is an update from a previously reported value of $4 \mathrm{kpc}$. We present the evidence that we have for the presence of oscillations at the pulsar frequency during part of the newly found burst. Such an oscillation would lend support to the idea that the frequency of millisecond burst oscillations in other objects is very close to the neutron star rotation frequency.
\end{abstract}

Key words. binaries: close - pulsars: individual (SAX J1808.4-3658) - X-rays: bursts

\section{Introduction}

SAX J1808.4-3658 was discovered with the Wide Field Cameras (WFCs) onboard the Italian-Dutch X-ray satellite BeppoSAX, in data taken during September 1996 (In 't Zand et al. 1998). Two intense type-I X-ray bursts were found which showed evidence of near-Eddington luminosities. Thus, a distance was estimated of $4 \mathrm{kpc}$, and the source was identified as a likely low-mass X-ray binary (LMXB). The outburst lasted approximately 3 weeks above a detection threshold of $10^{-10} \mathrm{erg} \mathrm{cm}^{-2} \mathrm{~s}^{-1}$ (2-10 keV), as measured with the All-Sky Monitor (ASM) on the Rossi X-ray Timing Explorer (RXTE).

The source was found with RXTE to be in outburst again in April-May 1998 (Marshall 1998). Firsttime observations with the Proportional Counter Array on RXTE showed a strong $401 \mathrm{~Hz}$ pulsar signal (Wijnands \& Van der Klis 1998). This represents the first and

Send offprint requests to: J. J. M. in 't Zand,

e-mail: jeanz@sron.nl

* Present address: University Hospital Nijmegen, Dept. of Radiology, Computer Aided Diagnosis, PO Box 9101, 6500 HB Nijmegen, The Netherlands.

** Present address: XMM-Newton Science Operations Center, Vilspa Satellite Tracking Station, Apartado 50727, 28080 Madrid, Spain. long-awaited unambiguous detection of an accreting millisecond pulsar. In the 2 years since this discovery, no other such pulsar has been found. Chakrabarty \& Morgan (1998) were able to determine orbital parameters from Doppler-shifted pulsations. The orbital period is $2.01 \mathrm{hr}$, and the mass function of the secondary star $3.8 \times 10^{-5} M_{\odot}$. Radio emission was detected once, at the time when the X-ray was about to disappear (Gaensler et al. 1999). The emission is attributed to ejection of material from the binary. It is believed that SAX J1808.4-3658 is the proof for a link between LMXBs and radio millisecond pulsars (e.g., Chakrabarty \& Morgan 1998). So far no pulsations have been found at radio wavelengths.

In January 2000, SAX J1808.4-3658 was found to be in outburst for a third time (Van der Klis et al. 2000). Unfortunately, the Sun was too close to the source to allow detailed coverage of the outburst. Nevertheless, the X-ray pulsar signal was again found.

Because of its unique nature, SAX J1808.4-3658 continues to be the subject of scientific studies. In this circumstance we considered it appropriate to revisit the WFC data of the first outburst. At the time of the first report by In 't Zand et al. (1998), the millisecond pulsar was not yet discovered and no high-resolution timing analysis was carried out on the WFC data. Furthermore, the coverage of the processing of $1996 \mathrm{WFC}$ data was incomplete. Also, 


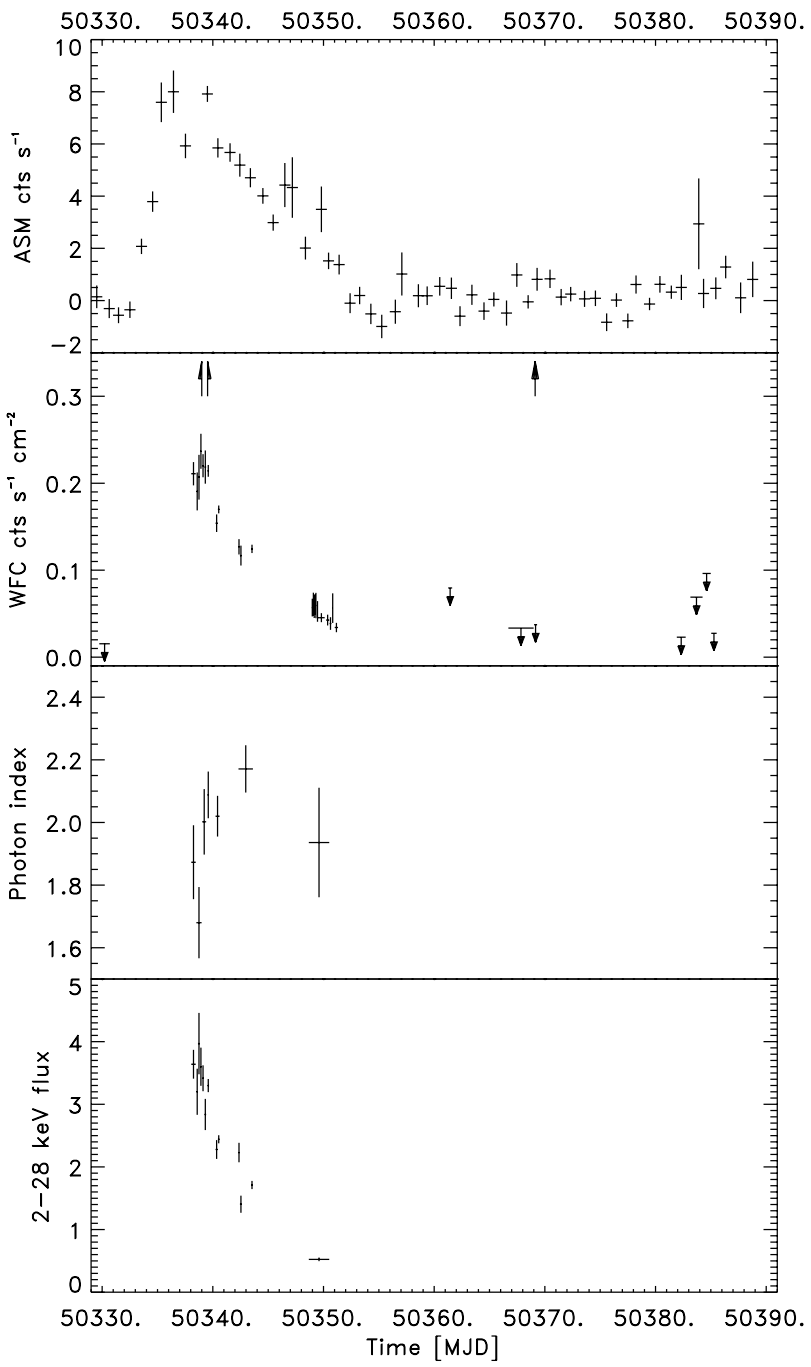

Fig. 1. Upper panel: ASM light curve of $2-12 \mathrm{keV}$ daily averages during the first outburst in 1996. In this time frame, there were 697 dwells over the source adding up to $63 \mathrm{ks}$. Second panel from above: WFC 2-28 keV light curve over the same period. The upward pointed arrows indicate the times of three X-ray bursts detected with the WFC. The downward pointed arrows indicate upper limits. The WFC exposure time in this time frame is $394 \mathrm{ks}$. For a Crab-like spectrum, $1 \mathrm{WFC} \mathrm{cts} \mathrm{s}^{-1} \mathrm{~cm}^{-2}$ is equivalent to $38 \mathrm{ASM} \mathrm{cts} \mathrm{s}^{-1}$. Third panel: photon index resulting from WFC spectral fits to persistent emission. Fourth panel: $2-28 \mathrm{keV}$ flux resulting from WFC spectral fits, in units of $10^{-9} \mathrm{erg} \mathrm{cm}^{-2} \mathrm{~s}^{-1}$.

analysis techniques and calibration of the WFCs have been updated to a more refined level. As a result of these improvements, it is now possible to obtain a more complete picture of SAX J1808.4-3658 during the first outburst. In this paper we present an analysis of "old" as well as "new" data and discuss the results. The main new results are the detection of a third type-I X-ray burst, the marginal detection of $401 \mathrm{~Hz}$ flux oscillations in that burst, and a refinement of the distance to SAX J1808.4-3658. We review the WFC observations in Sect. 2, discuss the data analysis in Sects. 3 through 6 , and discuss the implications in Sect. 7 .

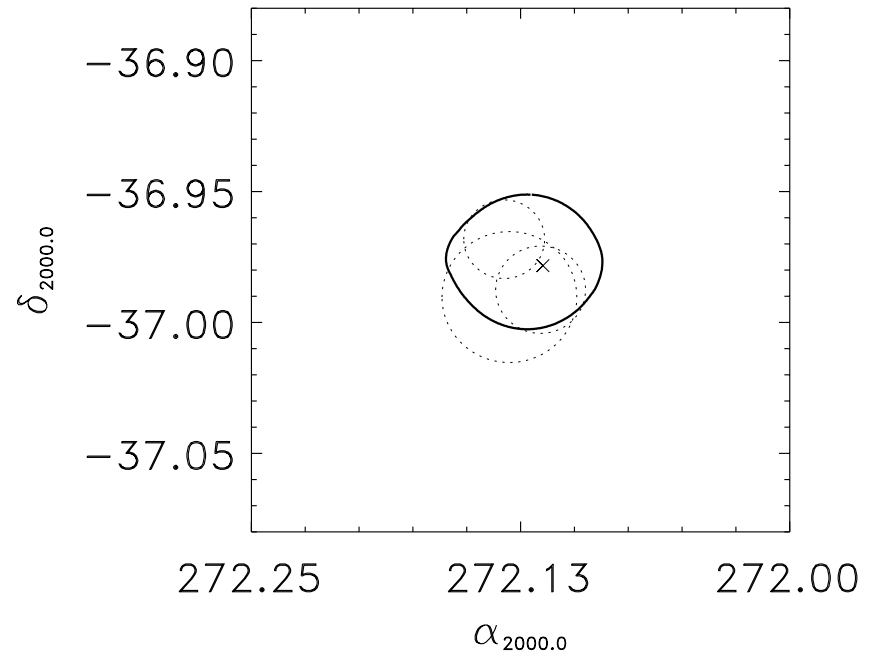

Fig. 2. Map of $99 \%$-confidence error regions of the 3 bursts (dotted circles) with respect to the optical counterpart (cross) and the error region of the persistent emission (thick loop).

\section{Observations}

The WFCs (Jager et al. 1997) are two identical coded aperture cameras, which were launched in April 1996 on board the BeppoSAX satellite (Boella et al. 1997). Each camera has a field of view of $40^{\circ} \times 40^{\circ}$ (full width to zero response), angular resolution of $5^{\prime}$ and is active between 2 and $28 \mathrm{keV}$. The on-axis detection threshold is of order a few mCrab in $10^{5} \mathrm{~s}$, and varies as a function of the total flux contained in the field of view. In 1996 the Galactic center was targeted by the WFCs for 22 days in the period August 21 to October 29. SAX J1808.4-3658 is 9.4 from the Galactic center, well within the field of view. The net exposure time for the source in 1996 is $550 \mathrm{ks}$.

Figure 1 presents the time history of the flux as measured with both WFC and ASM. The source was detected with the ASM above a threshold of about 0.02 Crab units for 22 days starting MJD 50333 (Sep. 5). The peak of 0.11 Crab units was reached within a few days. The WFC observations started while the source already was at the peak emission for about 3 days and carried on intermittently till 1 month past the moment when the source decayed below ASM detection levels. The sensitivity varied considerably due to varying off-axis angles over the different WFC observations. However, typical sensitivities compare to that of the ASM measurements.

Figure 1 also shows the times when X-ray bursts were detected. The first two bursts have already been reported in In 't Zand et al. (1998), the third one is for the first time reported here. The second burst follows quite quickly the first one $(0.5 \mathrm{~d})$, while the third burst follows 30 days later. Interestingly, the third burst occurred while no persistent emission was measured. If the persistent spectrum would have been similar to when the persistent flux was measured, the upper limit on the flux would be $3 \times 10^{-10} \mathrm{erg} \mathrm{cm}^{-2} \mathrm{~s}^{-1}$ (in $2-28 \mathrm{keV}$, for $10^{4} \mathrm{~s}$ exposures). 

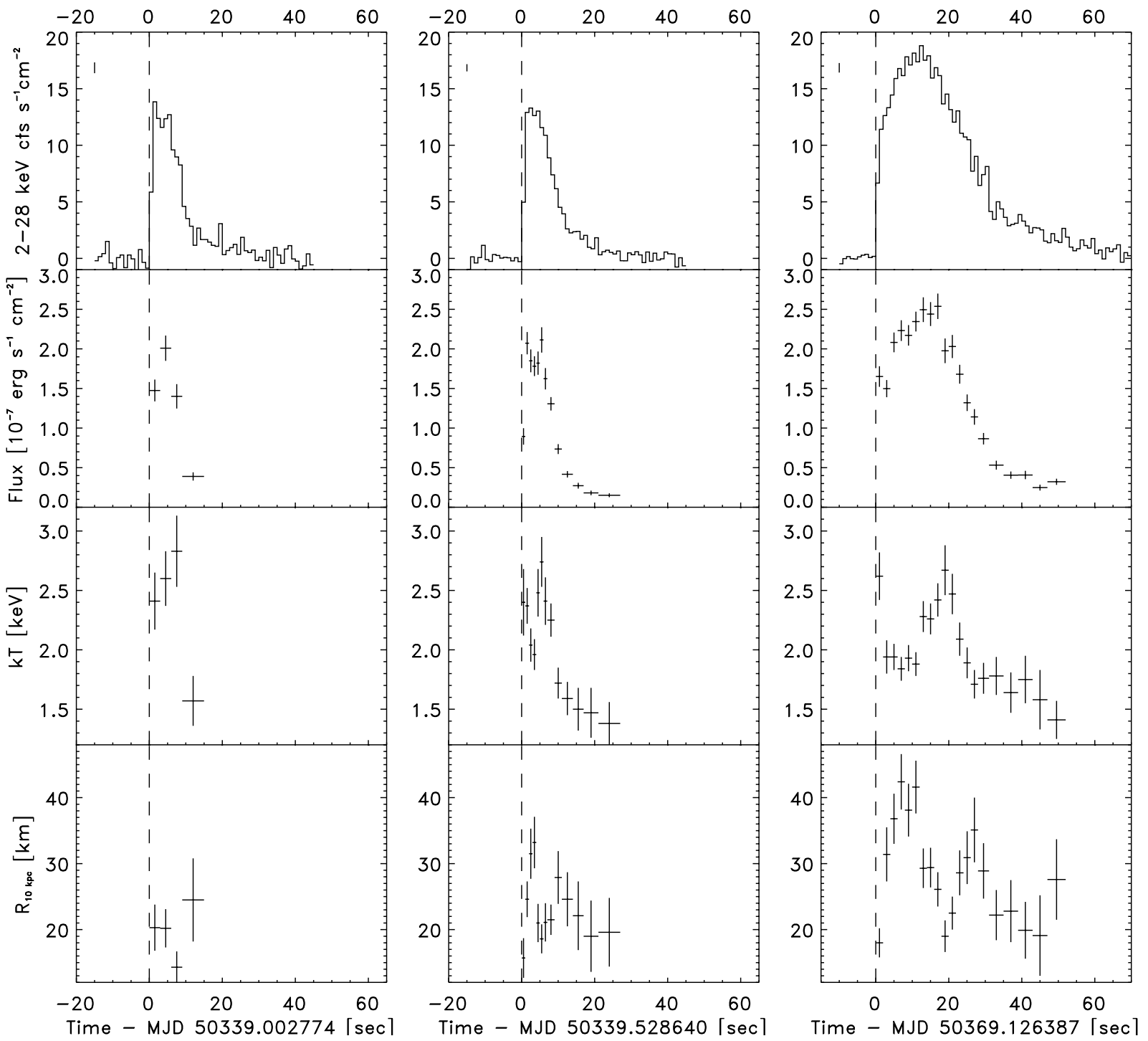

Fig. 3. Time profiles of photon fluxes (upper panel, with $1 \sigma$ error bars in the top left corner), bolometric flux (second panel), black-body color temperature (third panel) and black body radius (fourth panel) of black body radiation spectral fits, for the three bursts detected with WFC from SAX J1808.4-3658 during the 1996 outburst. The vertical dashed lines are drawn for guiding purposes and mark the onsets of each burst.

Figure 2 shows the error regions of all bursts with respect to that of the persistent emission and the optical counterpart (Giles et al. 1998). There is no doubt that these bursts are from SAX J1808.4-3658.

\section{Spectral analysis of the persistent emission}

The spectral data from the WFCs are read out in 31 channels between 2 and $28 \mathrm{keV}$. Three channels are not well calibrated and excluded in analyses. The SAX J1808.4-3658 detection data were resolved in 13 spectra. The data could successfully be modeled by a simple power-law function with varying photon index and a constant lowenergy absorption, parameterized by $N_{\mathrm{H}}$. For 7 different intervals, the resulting $\chi_{\mathrm{r}}^{2}=0.91$ (343 degrees of freedom). The photon index as a function of time is plotted in Fig. 1 (third panel). Although the data are not consistent with a constant photon index $\left(\chi_{\mathrm{r}}^{2}=\right.$ 1.21 for 349 dof), no clear trend is apparent. $N_{\mathrm{H}}$ was undetermined below an upper limit of $1 \times 10^{22} \mathrm{~cm}^{-2}$. The outburst peak fluxes as measured with the WFC are $4.0 \times$ $10^{-9} \mathrm{erg} \mathrm{cm}^{-2} \mathrm{~s}^{-1}(2-28 \mathrm{keV}), 2.1 \times 10^{-9} \mathrm{erg} \mathrm{cm}^{-2} \mathrm{~s}^{-1}$ $(2-10 \mathrm{keV})$ and $3.3 \times 10^{-9} \mathrm{erg} \mathrm{cm}^{-2} \mathrm{~s}^{-1}(3-25 \mathrm{keV})$.

\section{Spectral analysis of bursts}

We carried out time-resolved spectroscopy of the three bursts by modeling the 28-channel spectra with black body radiation. The time resolution is a compromise between good statistics to determine spectral parameters accurately enough, and time scales of spectral changes. We used resolutions between 1 and $7 \mathrm{~s}$. The results are presented in Fig. 3. A number of observations can be made:

- all bursts exhibit a fast rise and exponential-like decay; they are consistent with black body radiation, the color temperature peaks in all three cases at about $2.8 \mathrm{keV}$; and all bursts show cooling in the tail. These 
characteristics clearly suggest that these are thermonuclear flashes on the surface of a neutron star (see also In 't Zand et al. 1998);

- the second and third burst show variations in the extent of the emitting area and the corresponding color temperature, pointing to radius-expansion bursts due to luminosities near the Eddington limit. The asymptotic value for the extent of the emitting area, reached in the burst tails, is comparable for all three cases;

- the third burst lasts about three times as long as the others. In terms of peak brightness (9.3 Crab units in $2-28 \mathrm{keV}$ ) it is $50 \%$ brighter than the first two bursts. This makes the third burst the brightest type-I burst seen so far with the WFCs, out of a sample of 1700 cases. In terms of bolometric fluxes the difference decreases to $20 \%$ which is caused by a combination of a different color temperature during the peak and the limited bandpass of the instrument. If correcting for gravitational redshift effects, the difference disappears (see below);

- the bolometric fluences of the first two bursts are $2 \times$ $10^{-6}$ and that of the third burst is $7 \times 10^{-6} \mathrm{erg} \mathrm{cm}^{-2}$. If no other bursts occurred between the first two bursts, $\alpha$ (defined as the ratio of the fluence in the persistent emission between the bursts and that of the second burst) is $1.1 \times 10^{2}$. Given the ample WFC coverage, the wait time between bursts definitely is longer and $\alpha$ higher at later times when the third burst occurred.

\section{Distance}

The distance to the source can be estimated from the assumption that during the expansion and contraction phases the burst luminosity is close to the Eddington luminosity (see e.g. Lewin et al. 1993). In principle this is a simple method but in practice the uncertain value of the Eddington limit makes it less straightforward. The Eddington limit depends on the mass of the neutron star, on the electron scattering opacity (which changes with abundance and temperature), on the degree of isotropy and on how close the emission is to the neutron star (due to relativistic effects). The latter effect introduces a dependency on the relation between the color temperature to effective temperature. For a detailed discussion of these dependencies, we refer to Kuulkers et al. (2001).

We determined the distance to SAX J1808.4-3658 by employing the five highest flux measurements of the 2 nd burst and the seven highest of the 3rd burst. Assuming standard burst parameters (isotropy, cosmic abundances, $1.4 M_{\odot}$ neutron star mass, equivalency of the color to effective temperature, low-temperature opacity) we find distances of $2.57 \pm 0.15 \mathrm{kpc}$ and $2.37 \pm 0.12 \mathrm{kpc}$ for the $2 \mathrm{nd}$ and 3rd burst, respectively. If the actual color to effective temperature correction is $1 / 1.7$ instead of 1 , the distance would be about $10 \%$ larger; if the hydrogen abundance is not $X=0.73$ but $X=0.00$, the distance would be about $30 \%$ larger; if the neutron star mass is $2.0 M_{\odot}$, the distance would be about $20 \%$ larger. Therefore, we conclude that the distance is $2.5 \mathrm{kpc}$ but might just as well be $30 \%$ larger.

The $2.5 \mathrm{kpc}$ number is a revision of the $4 \mathrm{kpc}$ one reported by In 't Zand et al. (1998). This change is due to 1) application of the correction for gravitational redshift; 2) rounding the final number off after two digits instead of one; and 3) omitting the correction for detector dead time effects by In 't Zand et al. (1998). All 3 effects contribute at comparable levels. We note that the dead time correction was not omitted in later WFC papers.

\section{Search for oscillations}

In the telemetry data of the WFCs, each fourth event is labeled with a time stamp of accuracy $2^{-11} \mathrm{~s}$ and each 311 th event with one of accuracy $2^{-12} \mathrm{~s}$. This is sufficient resolution to search for a coherent pulsation near $401 \mathrm{~Hz}$. If the total event rate is of the same magnitude or higher, the other events (i.e., those without a time stamp) may also contribute to the sensitivity of such a search because Poisson fluctations in the rate may occasionally cause short enough wait times between two time stamps to enable accurate timing of the events in between. We carried out searches in two kinds of data: that of the bursts and that of the persistent emission during the brightest part of the transient outburst (on Sep. 12-13, 1996). The basis for the searches are Fourier power density spectra (PDS) of light curves generated at $2^{-11} \mathrm{~s}$ resolution from all photons that are detected in those parts of the detector that are illuminated by SAX J1808.4-3658 without being obstructed by the camera shielding or opaque parts of the mask. For the observations of SAX J1808.4-3658 outside the X-ray bursts, the total event rate on the detector is between 620 and $770 \mathrm{~s}^{-1}$. The portion of the detector area illuminated by SAX J1808.4-3658 varies between 6 and $24 \%$. During the first $20 \mathrm{~s}$ of the third burst, the average event rate is $1130 \mathrm{~s}^{-1}$ and the illuminated detector portion $12 \%$.

It is technically not feasible to generate lightcurves from reconstructed images of point sources at high time resolution. Therefore, we resort to taking photons directly. This implies that the flux from other sources within $20^{\circ}$ from SAX J1808.4-3658 along each image axis and within the field of view, or from the background, is not subtracted and that the timing signatures of those sources remain present in the data. However, since we are searching for a very specific feature at around $401 \mathrm{~Hz}$, this is not a major concern.

We barycentered the photon arrival times to the solar system barycenter and included a correction for the binary orbit using the ephemeris by Chakrabarty \& Morgan (1998) as determined from the 1998 outburst. This ephemeris is of sufficient accuracy to guarantee that the orbital phase during the 1996 outburst is accurate to $10^{-3}$.

The burst data lengths were $10 \mathrm{~s}$ for the first 2 bursts and $30 \mathrm{~s}$ for the third burst. While the first two bursts do not show any interesting signals, a small signal was 


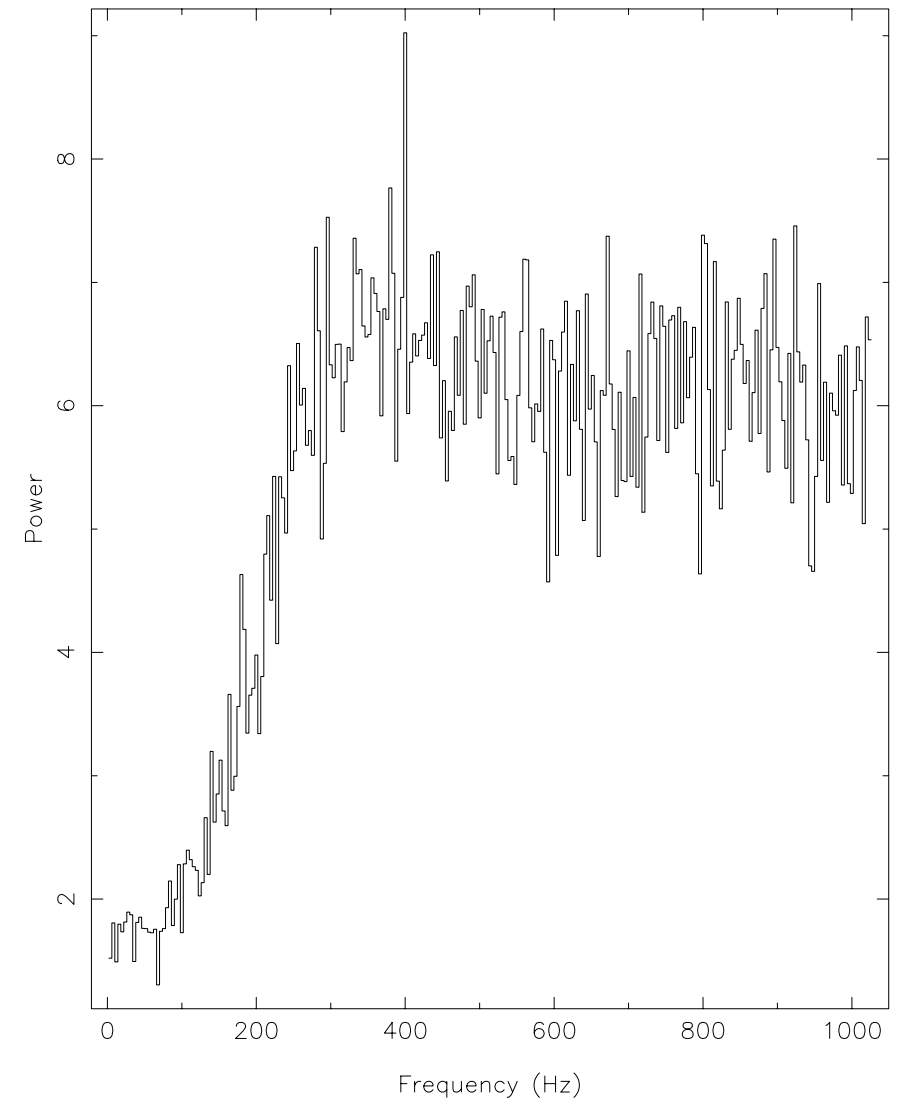

Fig. 4. PDS of the third burst. This is the average PDS of 80 data segments of $0.25 \mathrm{~s}$ duration. The trends in the background levels are due to the one-in-four event time labeling for WFC data, as was verified with simulations. The power is normalized following Leahy et al. (1983).

found near $401 \mathrm{~Hz}$ in one power spectrum of the third burst, namely for a frequency binning of $4 \mathrm{~Hz}$ (implied by a data segmenting in $0.25 \mathrm{~s}$ intervals), for the entire bandpass, and for averaging over the first $20 \mathrm{~s}$ of data, see Fig. 4. To assess the statistical significance of this feature, we evaluated the probability of measuring such a power or higher by chance through Monte Carlo (MC) calculations. We simulated the circumstances of the real observations, including the 1-in-4-events time stamping, the selection of the detector part and energy bandpass, the burst light curve (see Fig. 3) and an instrumental timing characteristic which causes a signal at $244 \mathrm{~Hz}$ and its harmonics. No $401 \mathrm{~Hz}$ periodic signal was included. The continua of the resulting power density spectra reproduce very well the observed one. Four out of 160000 MC simulations resulted in powers higher than the one observed. Thus, the chance probability for a single trial is $2.5 \times 10^{-5}$. This is equivalent to a $4.0 \sigma$ detection.

The number of trials is determined by the number of times that meaningful and independent searches for a burst oscillation have been carried out. This concerns three frequency binnings: 1,2 and $4 \mathrm{~Hz}$. Burst oscillations often show a frequency drift of a few $\mathrm{Hz}$ (for a review, see, e.g., Strohmayer 1999). Therefore, we regard searches with a finer resolution than $1 \mathrm{~Hz}$ as not meaningful. Searches are

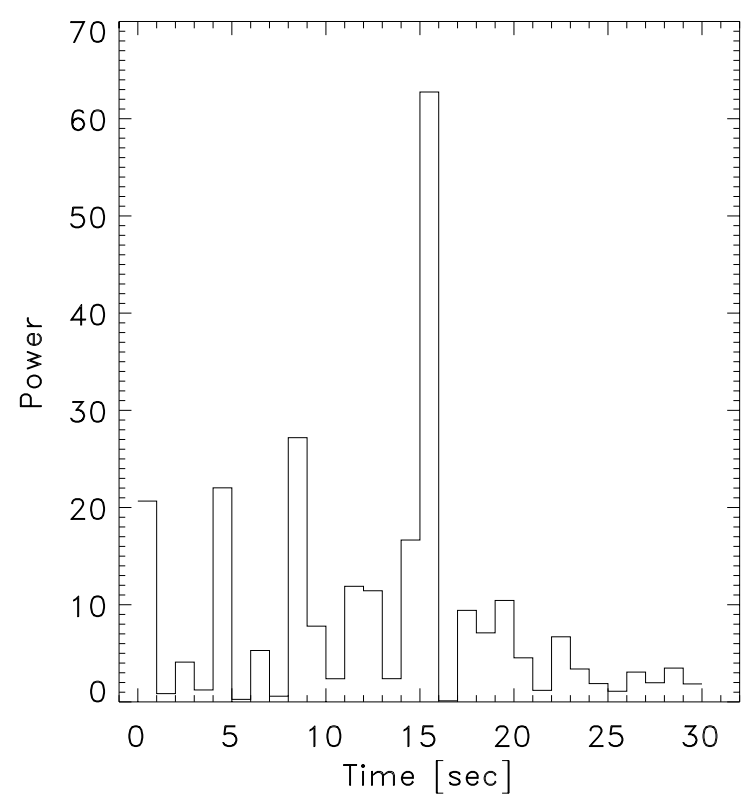

Fig. 5. Time history of power at $401 \mathrm{~Hz}$, at $1 \mathrm{~s}$ time resolution and $1 \mathrm{~Hz}$ frequency resolution. Time is in s since burst onset. Same data as used in Fig. 4. The chance probability for detecting the peak power or higher is $10^{-4}$ as was verified through MC calculations. The $10^{-2}$ chance probability level is at a power of about 30 .

only meaningful for the second and third burst because the statistical quality of the data of the first burst prohibits detecting even the highest amplitudes. Searches were done in two bandpasses, 2 to 10 and 10 to $28 \mathrm{keV}$ (and the total band, but this is a dependent trial). Finally, the sensitivity of a Fourier analysis that subdivides the data in a number of time series of equal length, and averages all resulting power spectra, is more sensitive when a number of different phases are used when subdividing for a particular length. We introduce 5 as the number of trials in this respect. In total, the number of trials is 60 . We note that in reality we carried out less trials to find the signal, and that we did search for signals at higher frequency resolution without positive results. The chance probability is $0.15 \%$ for 60 trials. This is equivalent to a $3.0 \sigma$ detection.

We tried to narrow down the time frame when the oscillation is present. This is difficult with such a weak signal; nevertheless, when cutting up the $20 \mathrm{~s}$ interval, the highest powers occur toward the end of the interval. At $1 \mathrm{~s}$ and $1 \mathrm{~Hz}$ resolutions, the peak occurs $15 \mathrm{~s}$ after the burst onset (see Fig. 5). This is immediately after the radius expansion phase, as is often seen in other burst oscillations.

The observed power is equivalent to an average oscillation amplitude of $60 \pm 6 \%$ of the source flux, as verified through simulations. If the signal actually comes from a smaller time interval, its duration is limited by a lower limit of $4 \mathrm{~s}$. If the duration would have been smaller, the implied amplitude would be larger than $100 \%$. 
The data of the persistent emission has an elapse time of $139 \mathrm{ks}$ and a net exposure time of $32 \mathrm{ks}$. In the resulting power density spectrum no coherent signal was found within $20 \mathrm{~Hz}$ from $401 \mathrm{~Hz}$. We also folded the data with the $400.9752106 \mathrm{~Hz}$ frequency as measured by Chakrabarty \& Morgan (1998) and again found no significant signal. The upper limit on the amplitude was determined by simulating the circumstances of the observations and fitting folded light curves with a sinusoid leaving free the amplitude and epoch. The $3 \sigma$ upper limit on the amplitude is about $20 \%$ of the source flux. This is a factor of 2 higher than the amplitude measured in the 1998 outburst (Wijnands \& Van der Klis 1998). Evidently, the pulsar signal is too weak to be picked up in the persistent emission.

\section{Discussion}

The evidence for $\sim 401 \mathrm{~Hz}$ burst oscillations in the third burst lends support to the idea that in other objects (with no pulsar signal) the frequency of burst oscillations may be identified with the neutron star rotation frequency. This is the first time that such direct evidence is presented for this idea. However, we point out that the evidence is marginal.

The revision of the distance has as most important implication that luminosities and intrinsic energy contents of outbursts are about 2.5 times less than previously thought. The peak $2-28 \mathrm{keV}$ luminosity of the first outburst is $3 \times 10^{36} \mathrm{erg} \mathrm{s}^{-1}$. If the spectrum had the same power law to $100 \mathrm{keV}$, like in the second outburst (Gilfanov et al. 1998), the bolometric peak luminosity may be of order $6 \times 10^{36} \mathrm{erg} \mathrm{s}^{-1}$, which is a few percent of the Eddington luminosity for a canonical 1.4 $M_{\odot}$ neutron star.

The third burst is different from the other two bursts, in terms of duration and fluence that are both about three times larger. The emitting area, as measured with $R_{10 \mathrm{kpc}}$ in the tail of the bursts, is the same for all 3 bursts. Theory predicts (for reviews, see Lewin et al. 1993; Bildsten 1998, 2000) that the duration and fluence of a type I X-ray burst are mainly determined by the atomic composition of the material being burned unstably during the burst, which is partly dependent on the stable nuclear reaction rate taking place before the burst which, in turn, depends on the accretion rate. The longer duration of the third burst suggests mixed hydrogen/helium burning, which would take place in a local accretion mass-flux regime of $\dot{m}<900 \mathrm{~g} \mathrm{~cm}^{-2} \mathrm{~s}^{-1}$ (Bildsten 2000). The persistent flux during the third burst is measured to be below $5 \times 10^{35} \mathrm{erg} \mathrm{s}^{-1}$. For a neutron star radius of $5 \mathrm{~km}$ (at $2.5 \mathrm{kpc}$ ), this translates to an upper limit to the mass flux rate of $9 \times 10^{2} \mathrm{~g} \mathrm{~cm}^{-2} \mathrm{~s}^{-1}$, which is consistent. The first two bursts are at mass accretion rates one order of magnitude above that limit, and are probably in the $\dot{m}>(2-5) \times 10^{3} \mathrm{~g} \mathrm{~cm}^{-2} \mathrm{~s}^{-1}$ regime of mixed hydrogen/helium bursts. This is confirmed by the value of $\alpha$.

Bursts were only seen in the first outburst. This can be explained as a selection effect. If we assume that each outburst lasts 2 months, the WFC observed the first outburst for $394 \mathrm{ks}$, the second outburst not at all, and the third outburst for $34 \mathrm{ks}$. RXTE did not observe the first outburst (except for $63 \mathrm{ks}$ with the ASM), the second outburst for $179 \mathrm{ks}$ and the third outburst for $146 \mathrm{ks}$. Ergo, the first outburst was covered the best.

Acknowledgements. We thank Michiel van der Klis and Fred Lamb for useful discussions, and Jaap Schuurmans and Gerrit Wiersma for data archive and software support. JZ and EK acknowledge financial support from the Netherlands Organization for Scientific Research (NWO). BeppoSAX is a joint Italian-Dutch program.

\section{References}

Bildsten, L. 1998, in The many faces of neutron stars, ed. R. Buccheri, J. van Paradijs, \& M. A. Alpar (Dordrecht: Kluwer), 419

Bildsten, L. 2000, in Cosmic Explosions, Proc. of the 10th Annual October Astrophysics Conf., ed. S. S. Holt, \& W. W. Zhang, in press [astro-ph/0001135]

Boella, G., Butler, R. C., Perola, G. C., et al. 1997, A\&AS, 122,299

Chakrabarty, D., \& Morgan, E. H. 1998, Nature, 394, 346

Gaensler, B. M., Stappers, B. W., \& Getts, T. J. 1999, ApJ, $522, \mathrm{~L} 117$

Giles, A. B., Hill, K. M., \& Greenhill, J. G. 1998, MNRAS, 304,47

Gilfanov, M., Revnivtsev, M., Sunyaev, R., \& Churazov, E. 1998, A\&A, 338, L83

In 't Zand, J. J. M., Heise, J., Muller, J. M., et al. 1998, A\&A, 331, L25

Jager, R., Mels, W. A., Brinkman, A. C., et al. 1997, A\&AS, 125,557

Kuulkers, E., Homan, J., Van der Klis, M., Lewin, W. H. G., \& Méndez, M. 2001, in preparation

Leahy, D. A., Darbro, W., Elsner, R. F., et al. 1983, ApJ, 266, 160

Lewin, W. H. G., van Paradijs, J., \& Taam, R. E. 1993, Space Sci. Rev., 62, 223

Marshall, F. E. 1998, IAUC, 6876

Strohmayer, T. E. 1999, in Proc. X-ray astronomy '999: Stellar endpoints, AGN, and the diffuse X-ray background, Astrophys. Lett. Comm., in press [astro-ph/9911338]

Van der Klis, M., Chakrabarty, D., Lee, J. C., et al. 2000, IAUC, 7358

Wijnands, R., \& van der Klis, M. 1998, Nature, 394, 344 